\title{
Variabilidad anatómica de los sistemas de conducción y estomático de genotipos de Prunus spp. de diferentes orígenes
}

\author{
Carlos Alberto Núñez-Colín ${ }^{(1)}$, Alejandro Facundo Barrientos-Priego(1), Juan Enrique Rodríguez-Pérez ${ }^{(1)}$ \\ y Raúl Nieto-Ángel(1)
}

\begin{abstract}
(1)Universidad Autónoma Chapingo, Instituto de Horticultura, Dep. de Fitotecnia, Km 38,5, Carretera México - Texcoco, 56230 Chapingo, Estado de México, México. E-mail: lit007@hotmail.com, abarrien@taurus1.chapingo.mx, jerodrig@taurus1.chapingo.mx, rnietoa@hotmail.com
\end{abstract}

\begin{abstract}
Resumen - El propósito de esta investigación fue estudiar las relaciones ambientales de disponibilidad de humedad, con las características anatómicas del sistema de conducción de agua y estomático, en cinco genotipos de duraznos mexicanos con diferentes orígenes (Jalatzingo y Misantla, Veracruz; Temascaltepec, México; Tulancingo, Oaxaca; Sombrerete, Zacatecas), un almendro y el portainjerto Nemaguard. Fueron caracterizados anatómicamente brotes, hojas y estomas de plántulas de seis meses de edad, mediante 25 caracteres. El almendro y el portainjerto Nemaguard presentaron diferencias con respecto a los duraznos, los cuales tuvieron mayor similitud, aunque mantuvieron una separación acorde con su origen. Los caracteres que diferenciaron los grupos fueron: densidad estomática, grosor de la epidermis superior, número, frecuencia y perímetro de vasos, el índice de vulnerabilidad de la nervadura central, y el porcentaje de médula, xilema y floema del brote. Las condiciones de disponibilidad de humedad del origen presentaron fuerte asociación con las características anatómicas estudiadas, debido a que los ambientes con mayor déficit de humedad (Tulancingo y Sombrerete) presentaron mayor tamaño y menor frecuencia de vasos de xilema, así como bajo porcentaje de xilema y floema en brote, lo que puede interpretarse como adaptaciones de resistencia a sequía. La zona donde es rara la ocurrencia de sequía (Jalatzingo) presentó características opuestas.
\end{abstract}

Términos para indexación: Prunus persica, Prunus dulcis, durazneros, almendro, xilema, estomas.

\section{Anatomical variability of water conduction and stomatal systems in genotypes of Prunus spp. of different origins}

\begin{abstract}
The aim of this research was to study the relationships between environmental humidity availability and the anatomical characteristics of water conduction and stomatal systems, in five Mexican peach genotypes with different origins (Jalatzingo and Misantla, Veracruz; Temascaltepec, México; Tulancingo, Oaxaca; Sombrerete, Zacatecas), one almond and Nemaguard rootstock. Shoots, leaves and stomata of six months old plants were anatomically characterized by means of 25 characters. The almond tree and the rootstock Nemaguard showed differences in relation to the peaches, which had higher similarity, although they maintained a separation according to their origin. Groups were differentiated by the following characters: stomatal density; width of the leaf upper epidermis; number, frequency and perimeter of xylem vessels; and the index of vulnerability of the leaf central vein, pith, xylem and phloem percentage of the shoot. The conditions of humidity availability of their origin showed strong association with the studied anatomical characteristics, because the environment with more deficit of humidity (Tulancingo and Sombrerete) showed plants with bigger size and smaller frequency of vessels of xylem, as well as low xylem and phloem percentage in shoot, which can be interpreted as resistance adaptations to drought. The area where the occurrence of drought (Jalatzingo) is rare showed the opposite characteristics.
\end{abstract}

Index terms: Prunus persica, Prunus dulcis, peaches, almond, xylem, stomata.

\section{Introducción}

Los cultivos frutícolas generalmente necesitan de suelos bien drenados, para lograr crecimiento y producción óptimos. El exceso de agua en el suelo puede causar daños o muerte a las plantas. Esta situación es más grave en suelos de textura pesada, con baja conductividad del agua, o en donde una capa impermeable al agua crea un manto freático elevado, durante periodos de excesiva humedad (Quamme \& Stushnoff, 1988). 
Las actuales variedades de duraznero (Prunus persica (L.) Batsch) son en su mayoría altamente homocigóticos, debido a que se trata de una especie autógama, generalmente autofecundadas por muchas generaciones. De aquí que la progenie derivada de estas variedades son poblaciones con características de planta y fruto muy similares a sus progenitores. A diferencia del caso anterior, los almendros (P. dulcis (L.) Batsch) generalmente presentan autoincompatibilidad por lo que, al ser alógamas obligadas, existe una gran heterogeneidad dentro de las poblaciones (Ryugo, 1993).

El durazno tiene una alta tasa de transpiración, comparado con otras especies frutales, pero esta transpiración depende de la permeabilidad cuticular al agua y las condiciones climáticas (Huguet et al., 1998). Además, se ha encontrado que las hojas son el sitio de mayor resistencia hidráulica, y en el caso del durazno existen evidencias que indican que el control estomático es pobre y que su xilema es altamente vulnerable (Améglio et al., 1998). La densidad estomática y el grosor de la epidermis son características que pueden estar relacionadas con la tolerancia a sequía (Baas, 1982), al ser las características anatómicas más expuestas de la planta, que representan el último eslabón del torrente transpiratorio hacia la atmósfera (Faust, 1989). Además, los portainjertos de durazno comúnmente usados son propagados mediante semilla (Hesse, 1993).

En contraste, el almendro es reportado como una de las especies frutales más resistentes a sequía, por su bajo flujo respiratorio (Kester \& Grasselly, 1987). La resistencia a sequía en durazno está caracterizada por un bajo flujo, tanto en xilema como en floema (Huguet et al., 1998). Por otra parte, Reyes-Santamaría et al. (2002) encontraron en aguacate presencia de vasos con diámetros menores, para aquellos genotipos que tienen una menor vulnerabilidad.

Las características anatómicas de tamaño de vasos y los porcentajes de xilema y floema, en tallos principales de frutales, son importantes para poder definir la cantidad de agua que pueda transportarse, ya que a medida que los porcentajes de estos tejidos son mayores y el diámetro de vasos menores, la cantidad de agua transportada será mayor, por lo tanto, habrá una mejor adaptación de las plantas a condiciones de alta humedad (Vasconcellos \& Castle, 1994; Reyes-Santamaría et al., 2002).

Debido a sus fuertes efectos, que enmascaran el verdadero valor de los genotipos, la heterogeneidad del ambiente y la interacción genotipo por ambiente han sido reconocidos como los dos más importantes factores que limitan la eficiencia en la evaluación genotípica. Con frecuencia, resulta que algunos individuos genéticamente pobres puedan resultar en fenotipos sobresalientes, debido a algunos factores ambientales positivos $\mathrm{y}$, por otra parte, que genotipos superiores puedan producir fenotipos mediocres, debido a efectos negativos de factores ambientales (Sahagún Castellanos, 1992).

Las variaciones adaptativas aumentan gradualmente su frecuencia al paso de las generaciones, a expensas de las menos adaptativas, este fenómeno es llamado selección natural, que es causa de la generación de individuos bien adaptados a su ambiente (Ayala \& Kieger, 1984).

En una población natural, que presenta adaptación a distintos ambientes, cambios ambientales fuertes son una probable causa de la pérdida de su adaptación, ya que algunas variantes genéticas, anteriormente favorables, pueden resultar desventajosas y pueden ser remplazadas por unas nuevas. En contraste, en poblaciones con alto grado de adaptación a ambientes específicos, la dotación genética ventajosa y desviaciones a partir de esta pueden ser inoportunas, en ciertos ambientes con condiciones diferentes. Una población específica posee caracteres que representan la acumulación de adaptaciones a un ambiente u origen, los cuales también deben ser reflejados a nivel de especie (Dobzhansky, 1970).

Los objetivos del presente estudio fueron evaluar las características anatómicas del sistema de conducción de agua y estomático, en cinco genotipos de durazno, un almendro y un portainjerto, su relación con el origen de eses grupos, y describir la variabilidad de estas características dentro y entre grupos.

\section{Material y Métodos}

El experimento se llevó a cabo en los invernaderos de fisiología vegetal, del Departamento de Fitotecnia de la Universidad Autónoma Chapingo, en Chapingo, Estado de México, ubicado a $19^{\circ} 29^{\prime} \mathrm{N}$ y a $98^{\circ} 53^{\prime} \mathrm{W}$, a una altitud de $2.240 \mathrm{~m}$.

Se evaluaron cuatro grupos de durazno y uno de almendro, obtenidos a partir de semillas de poblaciones de durazno "criollo", provenientes de Temascaltepec (Estado de México, México; $19^{\circ} 3^{\prime} \mathrm{N}$ y $100^{\circ} 3^{\prime} \mathrm{W}$ ); Misantla (Veracruz, México; 1956' N y 96 $50^{\circ} \mathrm{W}$ ); Sombrerete (Zacatecas, México; 2338' N y 103³8' W) y Tulancingo (Oaxaca, México; $17^{\circ} 45^{\prime} \mathrm{N}$ y $97^{\circ} 16^{\prime} \mathrm{W}$ ), y de una población de almendro (Prunus dulcis (L.) 
Batsch), provenientes del Banco Nacional de Germoplasma en España, de aproximadamente seis meses de edad. También se evaluaron plantas de duraznero, obtenidas en viveros comerciales, de la misma edad, una de población “criolla”, originaria de Jalatzingo (Veracruz, México; $19^{\circ} 48^{\prime} \mathrm{N}$ y $97^{\circ} 18^{\prime} \mathrm{W}$ ) y otra del portainjerto Nemaguard; todas establecidas en bolsas individuales, con un sustrato homogéneo de tierra de monte y arena, en proporción 1:1 en volumen.

Cinco plantas de cada grupo fueron evaluadas, de las cuales se obtuvo una impresión con silicón para huellas dentales (exactodent), en la cuarta hoja completamente extendida (primera hoja madura a partir del ápice); a esta impresión se obtuvo, mediante barniz de uñas, la impresión positiva y, después se fijó en un porta y cubreobjeto, para evaluar los caracteres de estomas. Se colectó el cuarto nudo y la quinta hoja completamente extendida, las que se fijaron en una solución FAA $\left(50 \%\right.$ etanol $96^{\circ}+5 \%$ ácido acético glacial + $10 \%$ formaldehído $40 \%$ de pureza $+35 \%$ agua), después se procesaron en un cambiador automático de tejidos (Tissuematon Fisher) con cellosolve y xileno, para transferirse a parafina $\left(55^{\circ} \mathrm{C}\right)$, donde permanecieron 72 horas dentro de una estufa. Se elaboró el taquete y pirámide de parafina de acuerdo con Sass (1968), en un micrótomo rotatorio (American Optical, modelo 820); se realizaron cortes transversales con grosor de $10 \mu \mathrm{m}$, en porta y cubreobjetos, mediante adhesivo Haupt y formol al 10\%, y su tinción se realizó con safranina y verde fijo, para después ser montados (Sass, 1968).

Los caracteres evaluados para estomas fueron a partir de diez campos (40X de objetivo y 10X de ocular), por impresión se determinó el número de estomas por $\mathrm{mm}^{2}$; la longitud de estomas $\left(10^{-2} \mathrm{~mm}\right)$ se cuantificó a tres estomas por campo, en un microscopio con una adaptación de una cámara de video de alta resolución, conectado a una tarjeta de captura de imágenes GrabIT Pro para su digitalización.

Los cortes transversales de brote fueron cuantificados en ocho campos por planta, basándose en el estudio de Sperry \& Saliendra (1994), quienes realizaron conteos cada $90^{\circ}$, por lo que se decidió hacer conteos cada $45^{\circ}$ para obtener: el número de vasos por $\mathrm{mm}^{2}$, el diámetro tangencial del vaso $\left(10^{-2} \mathrm{~mm}\right)$, área, perímetro y diámetro Feret $\left[(4 . a ́ r e a / \pi)^{0,5}\right]$, a 40X de objetivo y 10X de ocular, con el mismo equipo indicado anteriormente. El área y el porcentaje que cubre el xilema, floema, corteza y médula, a $2 \mathrm{X}$ de objetivo y $2,5 \mathrm{X}$ de ocular, en microscopio estereoscópico, con una adaptación a la cámara de video antes mencionada.

Se obtuvo la conductividad relativa del brote, mediante la fórmula Poiseuille modificada por Fahn et al. (1986), $\mathrm{CR}=\left(\mathrm{R}^{4}\right)(\mathrm{FRE})$, donde $\mathrm{CR}$ es la conductividad relativa, $\mathrm{R}$ es el radio del vaso y FRE es el número de vasos por $\mathrm{mm}^{2}$. También se calculó el índice de vulnerabilidad de Carlquist (1977), V = DV/FRE, donde $\mathrm{V}$ es la vulnerabilidad, DV es el diámetro del vaso y FRE es el número de vasos por $\mathrm{mm}^{2}$.

En cortes de hojas, se evaluó: el grosor de la lámina $\left(10^{-2} \mathrm{~mm}\right)$; el grosor de la epidermis superior e inferior $\left(10^{-2} \mathrm{~mm}\right)$, a $40 \mathrm{X}$ de objetivo y $10 \mathrm{X}$ de ocular; número de vasos por $\mathrm{mm}^{2} \mathrm{y}$ el diámetro tangencial, perímetro y área del vaso en la vena central, a 40X de objetivo y 10X de ocular; porcentaje de xilema en la vena central de la hoja, a 10X de objetivo y 10X de ocular, en el mismo equipo antes mencionado, y se obtuvo la conductividad relativa y la vulneribalidad de la vena central de la hoja con las mismas fórmulas que para brotes. La medición de caracteres a partir de imágenes digitalizadas se realizó mediante el programa Image Tool ver. 3.00 (Wilcox et al., 1995). De los datos obtenidos, se calculó los estadísticos básicos para ver el comportamiento de cada variable, dentro de cada grupo.

Se realizó un análisis en componentes principales (ACP), para observar la dispersión en un espacio bidimensional de los individuos de cada grupo, en los dos primeros componentes (Johnson, 1998). Con este análisis se representa, en forma gráfica, la variación existente entre los diferentes individuos de cada grupo, en $\mathrm{n}$ dimensiones, dependiendo de las $\mathrm{n}$ variables evaluadas, reduciendo la dimensionalidad al agrupar a todas las variables en componentes principales. De esta manera, es posible observar la similitud de los individuos de acuerdo a su cercanía (Durán Rodríguez \& Sahagún Castellanos, 1992).

La variabilidad intragrupo se determinó al obtener el centroide (coordenadas medias de los componentes principales) del grupo y al calcular la distancia euclidiana cuadrada de cada individuo, con respecto a su centroide, y comparar el promedio de estas distancias más menos su error estándar con los demás grupos (Núñez-Colín \& Barrientos-Priego, 2004). 
Se realizó un análisis discriminante canónico (ADC), para comparar los diferentes grupos y obtener variables que explican, de una mejor manera, la variabilidad entre grupos, así como la distancia de Mahalanobis para determinar si existen diferencias entre estos grupos (Johnson, 1998).

\section{Resultados y Discusión}

Con el propósito de explorar la homogeneidad dentro de cada grupo, con respecto a sus caracteres evaluados y con base en los coeficientes de variación (Cuadro 1), se determinó que el número de bolsas de metaxilema al aro principal del haz vascular es la característica más variable, dentro de los distintos grupos; solo los grupos durazno de Temascaltepec (México) y el portainjerto Nemaguard no presentan esta característica en los individuos evaluados. Así también, las variables conductividad relativa en la vena central de la hoja y la vulnerabilidad del brote son variables poco estables dentro de la mayoría de los grupos. Los grupos de Temascaltepec y Sombrerete presentaron solo una característica con un coeficiente de variación elevado, por lo que serían los más homogéneos.

El ACP se realizó, con el propósito de describir la variabilidad intragrupo con un enfoque multivariado. Así, los dos primeros componentes principales, con valores propios de 9,548 y 4,009, representaron 38,19 y 16,03\% de la variabilidad de los datos y lograron explicar el $54,23 \%$ de la varianza total.

Para determinar la dispersión existente dentro de cada grupo, con respecto a su centroide, y con base en la distancia euclidiana cuadrada promedio, se indica que valores altos representan alta variación y viceversa; así, el grupo con mayor dispersión fue el almendro, reflejado por el valor más alto, así como el máximo error estándar encontrado, que representa alta heterogeneidad de los individuos de este grupo; por el contrario los de menor dispersión fueron los de Temascaltepec y Sombrerete que fueron los más homogéneos (Figura 1). La existencia de una mayor dispersión es indicativo de una mayor heterogeneidad, lo cual es deseable en un programa de mejoramiento genético; por el contrario, al tener poca variabilidad y posiblemente llegar a una línea pura, permite homogeneizar la población, lo que provocaría utilizar técnicas genotécnicas de hibridación y no de selección; sin embargo, para la producción de portainjertos a nivel comercial esta es una gran ventaja, debido a su homogeneidad (Márquez Sánchez, 1985, 1988).

En el análisis discriminante canónico (ADC), con el uso de grupos como variable categórica, solamente fueron necesarias dos funciones canónicas (FC), para explicar la variabilidad existente entre grupos, las cuales acumulan el $85,17 \%$ de la varianza acumulada (Cuadro 2). La estructura canónica total en la primera función (Cuadro 3) detectó a las variables: densidad estomática y grosor de la epidermis superior, como las que diferenciaron, con mayor énfasis, a los grupos. Estas características están asociadas a la resistencia a sequía (Baas, 1982), que presenta cada grupo, y es posible asociarlas como adaptaciones adquiridas, para tolerar las condiciones ambientales prevalecientes. Además, al ser las estructuras anatómicas más expuestas del sistema de conducción de agua en la planta, son las que tienen más posibilidades de sufrir cambios adaptativos (Faust, 1989). Por lo anterior, altos valores en la primera FC identifican a genotipos con mayor grosor de epidermis superior y mayor densidad estomática; en el caso del almendro fueron los que menores valores tuvieron, caso contrario, fue el del portainjerto Nemaguard.

La segunda FC se asoció al comportamiento de las variables número y frecuencia de vasos, en la vena central de la hoja, así como el perímetro de estos y la vulnerabilidad en este mismo órgano y por el porcentaje que presentan de médula, xilema y floema en brote. Esta FC representó la capacidad del sistema de conducción para suministrar agua, está muy ligado al ambiente en el cual se desarrolló el genotipo. Así, altos valores de esta FC representan genotipos adaptados a condiciones de alta humedad, al tener mayor capacidad de conducción de agua (vasos pequeños y en alta densidad, así como altos porcentajes de xilema y floema en brote), y genotipos con bajos valores de esta FC indican que están adaptados a déficits hídricos (Vasconcellos \& Castle, 1994; Reyes-Santamaría et al., 2002)

La proyección de los grupos con base en las dos FC (Figura 2), en donde el almendro y Nemaguard se ubicaron en extremos opuestos en la FC1 (eje abscisas), mientras que los grupos de durazno se localizaron en el centro de este mismo eje, mostró que el almendro, una especie con alta resistencia a sequía (Kester \& Grasselly, 1987; Ryugo, 1993), presenta valores bajos en los 


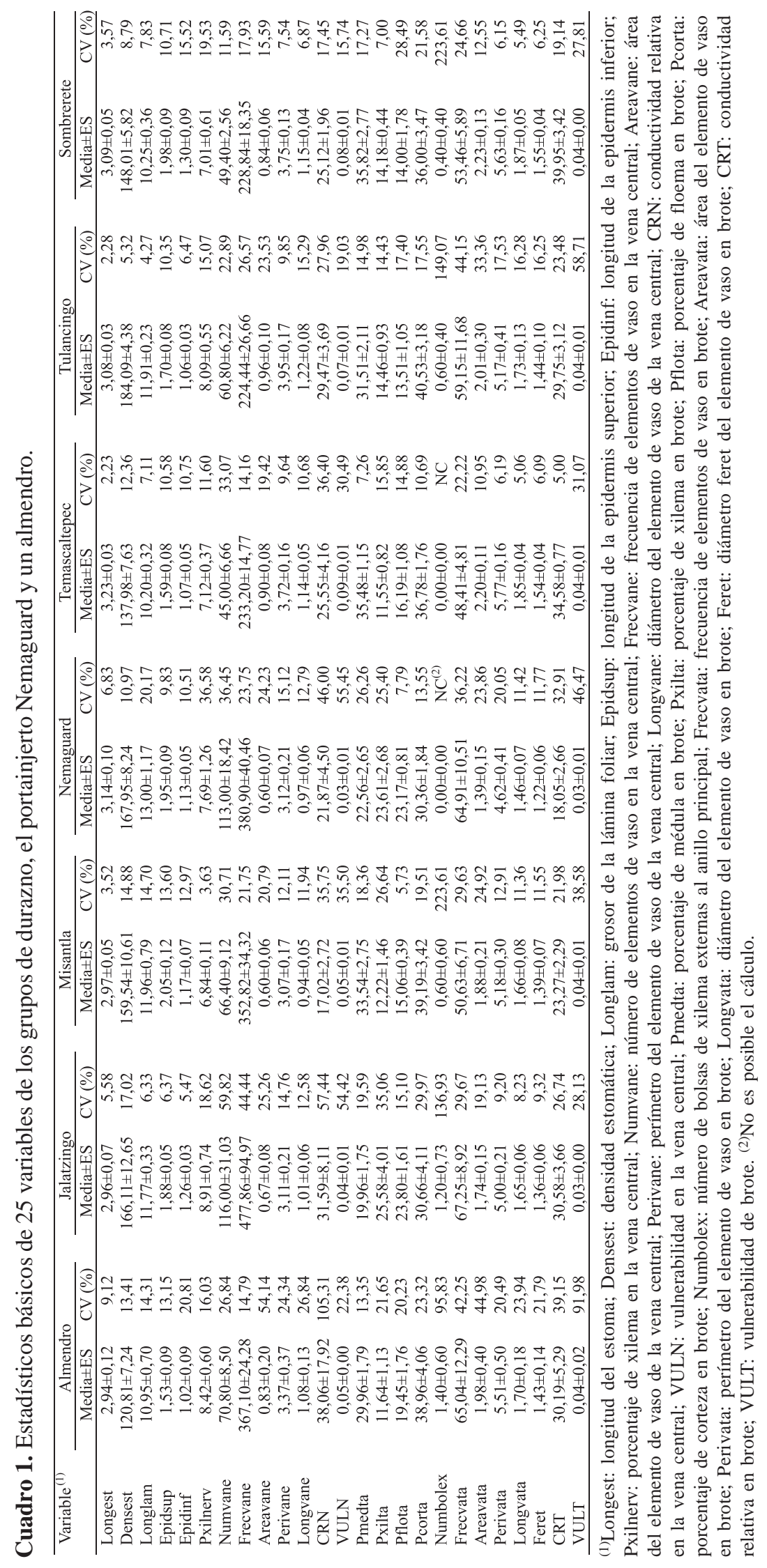


caracteres asociados a esta FC (densidad estomática y grosor de epidermis superior), que podrían estar relacionados con adaptaciones a condiciones de sequía; el caso contrario es el de Nemaguard, en el otro extremo del eje, que presenta altos valores de estos caracteres y es considerado como susceptible a sequía (Layne, 1987; Hesse, 1993; Ameglio et al., 1998).

La primer FC logró separar a las especies en estudio, de tal forma que el almendro se ubicó en el extremo negativo de esta FC, los duraznos en la parte media y Nemaguard, un híbrido interespecífico, en el extremo opuesto.

La separación de los grupos de durazno fue lograda mediante la segunda FC, y se mantuvo la integridad de

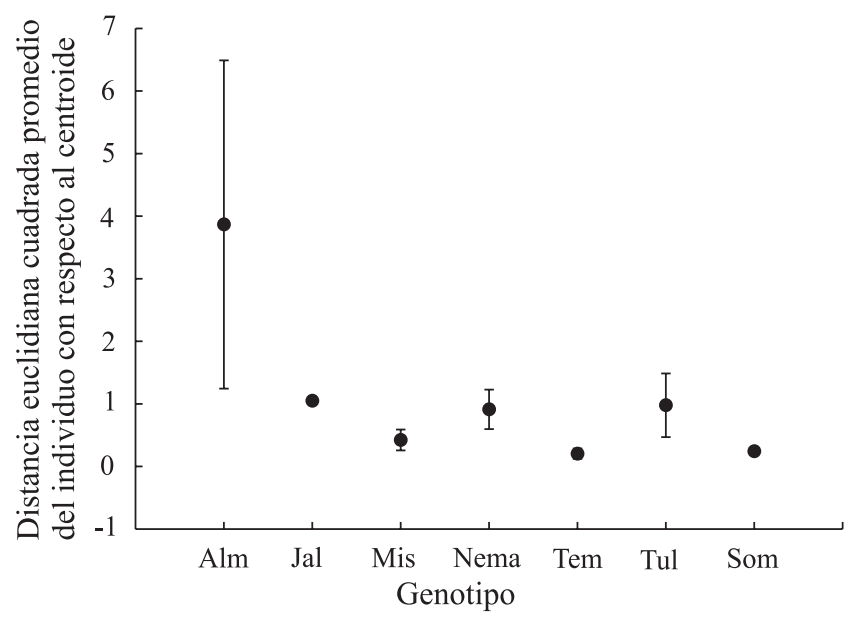

Figura 1. Variabilidad intragrupo del análisis en componentes principales, de grupos de duraznos provenientes de Jalatzingo (Jal), Misantla (Mis), Temascaltepec (Tem), Tulancingo (Tul) y Sombrerete (Som), el portainjerto Nemaguard (Nema) y un almendro (Alm), considerándose las características de brote, hoja y estomas. Distancia euclidiana cuadrada promedio de los individuos, con respecto a su centroide \pm error estándar.

Cuadro 2. Valores propios del análisis discriminante canónico de las variables anatómicas de brote, hojas y estomas de cinco grupos de durazno, el portainjerto Nemaguard y un almendro.

\begin{tabular}{ccccccc}
\hline $\begin{array}{c}\text { Función } \\
\text { canónica }\end{array}$ & $\begin{array}{c}\text { Valor } \\
\text { propio }\end{array}$ & $\begin{array}{c}\text { Varianza } \\
\text { explicada }\end{array}$ & $\begin{array}{c}\text { Varianza } \\
\text { acumulada }\end{array}$ & $\begin{array}{c}\text { Radio de } \\
\text { probabilidad }\end{array}$ & $\begin{array}{c}\text { F calculada } \\
\text { aproximada }\end{array}$ & $\mathrm{P}>\mathrm{F}$ \\
\hline 1 & 110,9300 & 0,5629 & 0,5629 & 0,00000011 & 3,01 & 0,0003 \\
2 & 56,9113 & 0,2888 & 0,8517 & 0,00001247 & 2,20 & 0,0077 \\
3 & 17,0142 & 0,0863 & 0,9381 & 0,00072217 & 1,49 & 0,1232 \\
4 & 8,1798 & 0,0415 & 0,9796 & 0,01300938 & 1,08 & 0,4360 \\
5 & 2,8462 & 0,0144 & 0,9940 & 0,11942359 & 0,72 & 0,8048 \\
6 & 1,1771 & 0,0060 & 1,0000 & 0,45932588 & 0,53 & 0,8865 \\
\hline
\end{tabular}

los orígenes. Así, los grupos provenientes de zonas con fuertes déficits hídricos fueron localizados con bajos valores en esta función, con las características de vasos grandes, pero en bajas densidades, así como bajas proporciones de xilema y floema, y altas de médula; esos caracteres favorecen un bajo flujo de agua en el sistema de conducción, y son mecanismos indicados como adaptaciones a resistencia a sequía (Vasconcellos \& Castle, 1994; Reyes-Santamaría et al., 2002); sin embargo, este tipo de anatomía adolece de la presencia de cavitaciones en sequías prolongadas (Baas, 1982).

Cuadro 3. Estructura canónica total (FC) y coeficientes canónicos totales (Can) del análisis discriminante canónico, de características anatómicas de brote, hoja y estomas de cinco grupos de durazno de diferentes procedencias, el portainjerto Nemaguard y almendro.

\begin{tabular}{lrrrr}
\hline Variable $^{(1)}$ & \multicolumn{1}{c}{ FC1 } & \multicolumn{1}{c}{ Can1 } & \multicolumn{1}{c}{ FC2 } & \multicolumn{1}{c}{ Can2 } \\
\hline Longest & 0,279793 & 0,10790039 & $-0,125351$ & $-0,17575856$ \\
Densest & 0,543969 & 3,68905125 & $-0,101884$ & $-2,39787656$ \\
Longlam & 0,295126 & $-2,07523089$ & 0,307780 & 0,53096429 \\
Epidsup & 0,531339 & 4,52084243 & 0,037400 & $-2,14862329$ \\
Epidinf & 0,278009 & 2,27014741 & $-0,030513$ & 1,95413895 \\
Pxilnerv & $-0,179315$ & 4,72830111 & 0,213656 & 1,14501537 \\
Numvane & 0,186529 & 11,04149476 & 0,563234 & 1,56408578 \\
Frecvane & $-0,060696$ & $-18,66851748$ & 0,610308 & $-4,26381776$ \\
Areavane & $-0,217060$ & $-11,09036009$ & $-0,383443$ & $-2,04027095$ \\
Perivane & $-0,057087$ & $-0,39235556$ & $-0,506347$ & $-0,66075293$ \\
Longvane & $-0,115143$ & $-4,92249671$ & $-0,406873$ & $-5,35166323$ \\
CRN & $-0,282246$ & 10,06084958 & 0,047193 & 4,01265606 \\
VULN & $-0,088373$ & 7,55491346 & $-0,622483$ & 1,03085844 \\
Pmedta & $-0,147776$ & $-48,31043235$ & $-0,644630$ & $-16,08943462$ \\
Pxilta & 0,394088 & $-44,09720084$ & 0,524575 & $-13,06061504$ \\
Pflota & 0,032194 & $-26,33968826$ & 0,802519 & $-5,05556866$ \\
Pcorta & $-0,240922$ & $-42,04580117$ & $-0,364550$ & $-14,55025534$ \\
Numbolex & $-0,343605$ & $-4,77121512$ & 0,086357 & $-1,22168941$ \\
Frecvata & $-0,051044$ & $-8,97937681$ & 0,241577 & $-1,41216408$ \\
Areavata & $-0,219730$ & 23,80344660 & $-0,408005$ & 3,13083317 \\
Perivata & $-0,268930$ & 0,02315638 & $-0,288270$ & $-0,77953645$ \\
Longvata & $-0,187033$ & 29,43779636 & $-0,415062$ & $-5,35430086$ \\
Feret & $-0,208022$ & $-43,99629356$ & $-0,425654$ & 3,42101696 \\
CRT & $-0,251608$ & $-4,93039213$ & $-0,438415$ & $-2,37530699$ \\
VULT & $-0,139921$ & $-17,99909809$ & $-0,238707$ & $-0,73300737$ \\
\hline
\end{tabular}

(1)Longest: longitud del estoma; Densest: densidad estomática; Longlam: grosor de la lámina foliar; Epidsup: longitud de la epidermis superior; Epidinf: longitud de la epidermis inferior; Pxilnerv: porcentaje de xilema en la vena central; Numvane: número de elementos de vaso en la vena central; Frecvane: frecuencia de elementos de vaso en la vena central; Areavane: área del elemento de vaso de la vena central; Perivane: perímetro del elemento de vaso de la vena central; Longvane: diámetro del elemento de vaso de la vena central; CRN: conductividad relativa en la vena central; VULN: vulnerabilidad en la vena central; Pmedta: porcentaje de médula en brote; Pxilta: porcentaje de xilema en brote; Pflota: porcentaje de floema en brote; Pcorta: porcentaje de corteza en brote; Numbolex: número de bolsas de xilema externas al anillo principal; Frecvata: frecuencia de elementos de vaso en brote; Areavata: área del elemento de vaso en brote; Perivata: perímetro del elemento de vaso en brote; Longvata: diámetro del elemento de vaso en brote; Feret: diámetro feret del elemento de vaso en brote; CRT: conductividad relativa en brote; VULT: vulnerabilidad de brote. 
Las probabilidades de la distancia de Mahalanobis (Cuadro 4) indicaron que el grupo de almendro presentó diferencias significativas con todos los duraznos, esto es debido a que son especies diferentes. Nemaguard, al ser un híbrido interespecífico entre P. persica (L.) Batsch x P. davidiana (Carr.) Franch, presentó diferencias significativas con los grupos de duraznero, excepto con los grupos de las zonas más húmedas, por lo que pueden compartir la susceptibilidad a sequía indicada por Hesse (1993). El grupo de Jalatzingo presenta la máxima distancia entre durazneros, de los

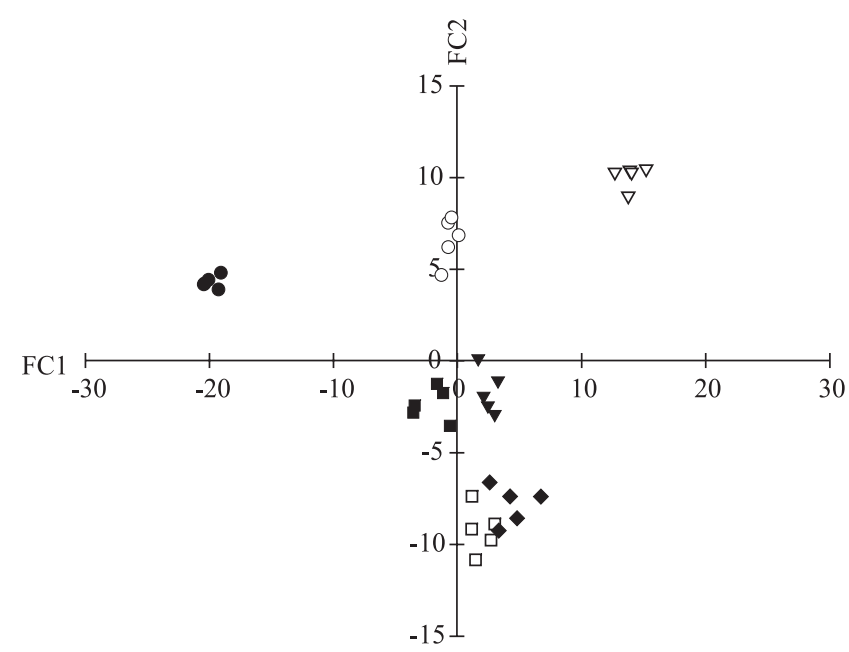

Figura 2. Dispersión de los cinco grupos de durazno, Nemaguard y almendro, en el primer plano factorial de las dos primeras funciones canónicas del análisis discriminante canónico, derivadas de características anatómicas de brote, hojas y estomas. FC1: densidad estomática y grosor de la epidermis superior. FC2: número, frecuencia y perímetro de vasos, vulnerabilidad en la nervadura central, y los porcentajes de médula, xilema y floema del brote. Almendro: ; Jalatzingo: $\bigcirc$; Misantla: $\mathbf{\nabla}$; Nemaguard: $\nabla$; Temascaltepec: $\mathbf{\square}$; Tulancingo: $\square$; Sombrerete: grupos de Sombrerete y de Tulancingo, aunque estadísticamente no son diferentes. Son grupos de zonas contrastantes, ya que estos dos últimos provienen de zonas áridas de México y, probablemente, son los más tolerantes a sequía; el grupo de Jalatzingo proviene de la zona más húmeda, por lo tanto se asume que es el menos tolerante a sequía, lo que se puede confirmar por las características del tamaño de elementos de vaso y la frecuencia de estos en la vena central de la hoja, la vulnerabilidad en la vena central de hoja y los porcentajes del xilema, floema y médula del brote, en el sistema de conducción de agua (Sánchez-Díaz \& Aguirreolea, 2000).

Los grupos de Sombrerete, Tulancingo, Temascaltepec y Misantla no presentaron diferencias significativas entre si, porque estos grupos provienen de zonas donde existen problemas de abastecimiento de agua, por lo menos en alguna parte del año.

La agrupación coincide tanto con el origen geográfico como con la constitución genética de los grupos estudiados (Figura 2). El almendro, al ser una especie distinta y poseer diferencias anatómicas y morfológicas marcadas con el durazno, fue separado de forma clara. Esta diferencia puede ser corroborada por la distancia de Mahalanobis, las cuales indican diferencias significativas con el resto de los grupos; situación similar ocurrió con el híbrido Nemaguard.

Los resultados anteriores dan mayor claridad sobre aspectos de adaptación de los genotipos estudiados, sin embargo, hay que tener en cuenta las relaciones existentes entre la parte aérea y raíz, que son de gran importancia (Itipps et al., 1995), ya que se ha

Cuadro 4. Probabilidades y distancias de Mahalanobis, de características anatómicas de brote, hoja y estomas en cinco grupos de durazno de diferentes procedencias, el portainjerto Nemaguard y almendro.

\begin{tabular}{|c|c|c|c|c|c|c|c|}
\hline Genotipo & Almendro & Jalatzingo & Misantla & Nemaguard & Tulancingo & Sombrerete & Temascaltepec \\
\hline Almendro & - & 445,47 & 555,40 & 1175,00 & 695,74 & 755,26 & 390,56 \\
\hline Jalatzingo & $0,04^{*}$ & - & 212,46 & 304,84 & 295,09 & 291,77 & 195,64 \\
\hline Misantla & $0,03^{*}$ & 0,14 & - & 291,09 & 143,93 & 100,23 & 75,70 \\
\hline Nemaguard & $0,01^{* *}$ & 0,09 & 0,09 & - & 560,46 & 440,28 & 435,94 \\
\hline Tulancingo & $0,02^{*}$ & 0,09 & 0,25 & $0,03^{*}$ & - & 89,77 & 169,61 \\
\hline Sombrerete & $0,02^{*}$ & 0,09 & 0,40 & $0,04^{*}$ & 0,45 & - & 100,55 \\
\hline Temascaltepec & $0,05^{*}$ & 0,16 & 0,53 & $0,04^{*}$ & 0,20 & 0,40 & - \\
\hline
\end{tabular}

${ }^{*} \mathrm{y}{ }^{* *}$ Significativo a 5 y $1 \%$ de probabilidad, respectivamente. 
comprobado que existen diferencias en cuanto a resistencia a sequía, al utilizar cultivares en sus propias raíces, en comparación con plantas injertadas en portainjertos provenientes de semillas, donde estas últimas fueron más susceptibles (Couvillon et al., 1989).

\section{Conclusiones}

1. Las características anatómicas, evaluadas en el presente estudio, están fuertemente relacionadas con las condiciones ambientales de abastecimiento de humedad, del lugar de origen de los grupos de durazno.

2. Los grupos de durazno se agrupan de acuerdo a las condiciones de su lugar de origen, quedando juntos: Sombrerete y Tulancingo, de zonas semiáridas con poca disponibilidad de agua; Misantla y Temascaltepec, de zonas templadas subhúmedas y Jalatzingo de una zona subtropical húmeda.

3. El almendro presenta alta heterogeneidad; el portainjerto Nemaguard presenta heterogeneidad moderada de sus características, pero por el linaje de P. davidiana (Carr.) Franch presenta diferencias con los duraznos.

4. Los grupos de durazno presentan poca heterogeneidad de características y los grupos de Sombrerete y Temascaltepec son los más homogéneos.

\section{Agradecimientos}

Al Consejo Nacional de Ciencia y Tecnología (CONACYT, México), por la concesión de beca; a la Dirección General de Investigación y Posgrado de la Universidad Autónoma Chapingo.

\section{Referencias}

AMÉGLIO, T.; COCHARD, H.; PICON, C.; COHEN, M. Water relations and hydraulic architecture of peach trees under drought conditions. Acta Horticulturae, v.465, p.355-362, 1998.

AYALA, F.J.; KIEGER JUNIOR, J.A. Genética moderna. Barcelona: Editorial Fondo Educativo Interamericano, 1984. 836p.

BAAS, P. Systematic phylogenetic and ecological wood anatomy: history and perspectives. In: BAAS, P. (Ed.). New perspectives in wood anatomy. The Hague: M. Nijhoff, 1982. p.23-58.
CARLQUIST, S. Ecological factors in wood evolution: a floristic approach. American Journal of Botany, v.64, p.887-896, 1977.

COUVILLON, G.A.; RIEGER, M.; APARISI, J.; HARRISON, R.; DANIELL, J. Stress-mediated responses of own rooted peach cultivars. Acta Horticulturae, v.243, p.221-230, 1989.

DOBZHANSKY, T. Genetics of the evolutionary process. New York: Columbia University Press, 1970. 505p.

DURÁN RODRÍGUEZ, R.; SAHAGÚN CASTELLANOS, J. La interacción genético-ambiental en la genotecnia vegetal. Revista Chapingo, v.16, p.79-80, 1992.

FAHN, A.; WERKER, E.; BAAS, P. Wood anatomy and identification of trees and shrubs from Israel and adjacent regions. Jerusalem: Israel Academy of Sciences and Humanities, 1986. 221p.

FAUST, M. Physiology of temperate zone fruit trees. New York: Wiley, 1989. 338p.

HESSE, C.O. Duraznos. In: MOORE, J.N.Y.; JANICK, J. (Ed.). Avances en la genotecnia de frutales. México: AGT Editor, 1993. p.359-424.

HIPPS, N.A.; PAGÈS, L.; HUGUET, J.G.; SERRA, V. Influence of controlled water supply on shoot and root development of young peach trees. Tree Physiology, v.15, p.95-103, 1995.

HUGUET, J.G.; GÉNARD, M.; LAURENT, R.; BESSET, J.; BUSSI, C.; GIRARD, T. Xylemic, phloemic and transpiration flows to and from a peach. Acta Horticulturae, v.465, p.345-354, 1998.

JOHNSON, D.E. Métodos multivariados aplicados al análisis de datos. México: International Thomson Editores, 1998. 590p.

KESTER, D.E.; GRASSELLY, C. Almond rootstocks. In: ROM, R.C.; CARLSON, R.F. (Ed.). Rootstocks for fruit crops. New York: Wiley, 1987. p.265-293.

LAYNE, R.E.C. Peach rootstocks. In: ROM, R.C.; CARLSON, R.F. (Ed.). Rootstocks for fruit crops. New York: Wiley, 1987. p.185-216.

MÁRQUEZ SÁNCHEZ, F. Genotecnia vegetal: métodos, teoría y resultados: tomo I. México: AGT, 1985. 356p.

MÁRQUEZ SÁNCHEZ, F. Genotecnia vegetal: tomo II. México: AGT, 1988. 563p.

NÚÑEZ-COLÍN, C.A.; BARRIENTOS-PRIEGO, A.F. Variabilidad interna, un uso alternativo del análisis en componentes principales. Proceedings of the Interamerican Society for Tropical Horticulture, v.48, p.203-207, 2004.

QUAMME, H.A.; STUSHNOFF, C. Resistencia al estrés ocasionado por el medio ambiente. In: MOORE, J.N.Y.; JANICK, J. (Ed.). Métodos genotécnicos en frutales. México: AGT, 1988. p.323-355. 
REYES-SANTAMARÍA, I.; TERRAZAS, T.; BARRIENTOSPRIEGO, A.F.; TREJO, C. Xylem conductivity and vulnerability in cultivars and races of avocado. Scientia Horticulturae, v.92, p.97-105, 2002.

RYUGO, K. Fruticultura, ciencia y arte. México: AGT, 1993. 460p.

SAHAGÚN CASTELLANOS, J. El ambiente, el genotipo y su interacción. Revista Chapingo, v.16, p.5-12, 1992.

SÁNCHEZ-DÍAZ, M.; AGUIRREOLEA, J. Trasporte de agua y balance hídrico en la planta. In: AZCÓN-BIETO, J.; TALÓN, M. (Ed.). Fundamentos de fisiología vegetal. Barcelona: McGrawHill Interamericana, 2000. p.45-64.
SASS, J.E. Botanical microtechnique. $3^{\text {rd }}$ ed. Ames: The Iowa State University Press, 1968. 227p.

SPERRY, J.S.; SALIENDRA, N.Z. Intra and inter-plant variation in xylem cavitation in Betula occidentalis. Plant, Cell and Environment, v.17, p.1233-1241, 1994.

VASCONCELLOS, L.A.B.C.; CASTLE, W.S. Trunk xylem anatomy of mature healthy and blighted grapefruit trees on several rootstocks. Journal of the American Society for Horticultural Science, v.119, p.185-194, 1994.

WILCOX, D.; DOVE, B.; McDAVID, D.; GREER, D. Image tool, version 3: user's guide. San Antonio: University of Texas Health Science Center, 1995. 62p.

Recibido el 31 de mayo de 2004 y aceptado el 29 de junio de 2005 